\title{
Defect production by pure phase twist injection as Aharonov-Bohm effect
}

\author{
Matteo Foresti* \\ Department of Mathematics \& Applications, U. Milano-Bicocca, 20125 Milano, Italy \\ Renzo L. Ricca ${ }^{\dagger}{ }^{\dagger}$ \\ Department of Mathematics \& Applications, U. Milano-Bicocca, 20125 Milano, Italy \\ and BDIC, Beijing U. Technology, 100 Pingleyuan, Beijing 100124, People's Republic of China
}

(Received 30 January 2019; revised manuscript received 19 June 2019; published 16 August 2019)

\begin{abstract}
In this paper we demonstrate that new phase defects of the Gross-Pitaevskii equation (GPE) can be produced as a Aharonov-Bohm effect resulting from pure phase twist injection on existing defects. This is a phenomenon that has physical justification in the hydrodynamic interpretation of GPE. Here we give an analytical proof of its effects by using Fermi-Walker transport and Biot-Savart induction law. An analytical derivation of the dispersion relation is derived from the superposition of phase twist on the fundamental state. Since the extra twist corresponds to a topological change of the total linking number of the system, we show that the production of new defects is just another manifestation of the Aharonov-Bohm effect. We propose a laboratory experiment for Bose-Einstein condensates to test this phenomenon and to show that it can have useful applications in science and technology.
\end{abstract}

DOI: 10.1103/PhysRevE.100.023107

\section{INTRODUCTION}

In recent papers Zuccher and Ricca [1,2] examined the effect of twist superposition on the dynamics and interaction of phase defects (quantum vortices) under the three-dimensional (3D) Gross-Pitaevskii equation. Phase twist, defined in terms of the rotation of the isophase surface of the wave function, was shown to have physical, measurable effects in terms of the production of new defects [2], but no rigorous proof was given. Here we demonstrate analytically that indeed the superposition of an isophase twist determine the production of new defects. Spontaneous generation of new defects through phase twist induction is an interesting, novel phenomenon that deserves further attention for its potential in theory and applications. In this paper we provide an analytical proof and a physical explanation of the direct relationship between extra twist and production of new defects in Bose-Einstein condensates (BECs). For this let us consider the 3D GrossPitaevskii equation (GPE) $[3,4]$, given by

$$
i \hbar \frac{\partial \Psi}{\partial t}=-\frac{\hbar^{2}}{2 m_{0}} \nabla^{2} \Psi+\left(g|\Psi|^{2}-\frac{1}{2}\right) \Psi,
$$

where $i=\sqrt{-1}, \hbar=h / 2 \pi$ ( $h$ Planck's constant), $m_{0}$ is the boson's mass, $g$ the coupling constant, $\Psi=\Psi(\mathbf{x}, t)$ the complex wave function and $\mathbf{x}$ denotes the position vector of a point in the ambient space and $t$ time. By standard rescaling [5] the equation above reduces to the nondimensional GPE in standard form, given by

$$
\frac{\partial \psi}{\partial t}=\frac{i}{2} \nabla^{2} \psi+\frac{i}{2}\left(1-|\psi|^{2}\right) \psi
$$

\footnotetext{
*matteo.foresti@unimib.it
}

†renzo.ricca@unimib.it for the complex wave function $\psi=\psi(\mathbf{x}, t)$, assuming background unit density $\rho=|\psi|^{2} \rightarrow 1$ as $|\mathbf{x}| \rightarrow \infty$. In this context defects emerge as singularities of the wave function $\psi$ (nodal lines). By using the Madelung transformation $\psi=$ $\sqrt{\rho} \exp (i \chi)$, where $\chi$ (action) denotes the phase of $\psi$, the real and imaginary parts of (2) are mapped to a momentum and a continuity equation of a fluidlike medium of density $\rho$ and velocity $\mathbf{u}=\nabla \chi$, allowing a macroscopic interpretation of GPE in terms of standard hydrodynamics [5]. In this context a phase defect is identified with a vortex line given by a closed space curve $\mathcal{L}$ of total length $L$ and quantized circulation (per unit of mass) $\Gamma=2 \pi n(n=1,2, \ldots)$ that represents the topological charge of the vortex. Quantization of circulation arises from the line integration of $\mathbf{u}=\nabla \chi$ over a simple loop encircling $\mathcal{L}$ and the multivaluedness of $\chi$. Alternatively, $\Gamma=2 \pi n$ can be regarded as the strength of a star-type defect of $n$ wavefronts departing from the singularity line [6] - a case also well exemplified by the multiarms scroll waves observed in reaction-diffusion systems of excitable media [7]. Vorticity $\omega$ is singular on $\mathcal{L}$, being everywhere zero except on the vortex line.

Quantum vortices move in space according to Eulerian dynamics [8] and the relationship between vorticity $\boldsymbol{\omega}$ at $\mathbf{X}^{*}=$ $\mathbf{X}^{*}(s)$ ( $s$ arc-length on $\mathcal{L}$ ) and induced velocity $\mathbf{u}$ at $\mathbf{x} \neq \mathbf{X}^{*}$ is thus governed by the Biot-Savart induction law, given by

$$
\mathbf{u}(\mathbf{x})=\frac{\Gamma}{4 \pi} \oint_{\mathcal{L}} \frac{\hat{\mathbf{T}}\left(\mathbf{X}^{*}\right) \times\left(\mathbf{x}-\mathbf{X}^{*}\right)}{\left|\mathbf{x}-\mathbf{X}^{*}\right|^{3}} d s,
$$

where $\hat{\mathbf{T}}\left(\mathbf{X}^{*}\right)$ denotes the unit tangent to $\mathcal{L}$ at $\mathbf{X}^{*}$. The integral above is a global geometric functional of vorticity with effects on the induced velocity field $\mathbf{u}$. Kinetic helicity, a conserved 


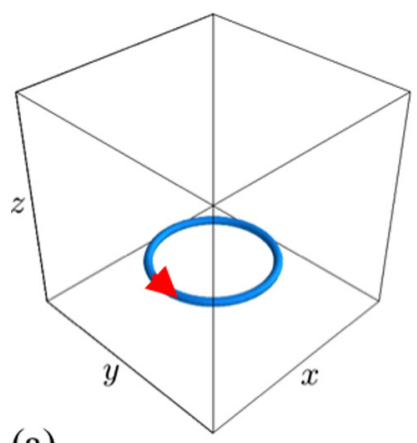

(a)

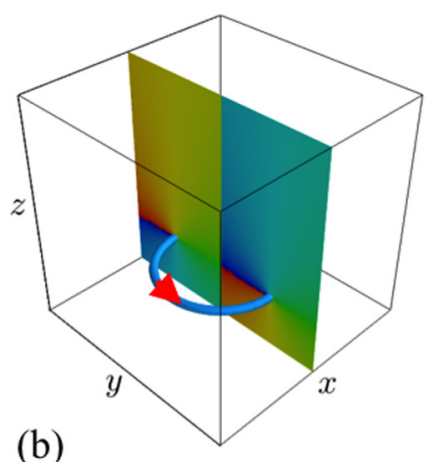

(b)

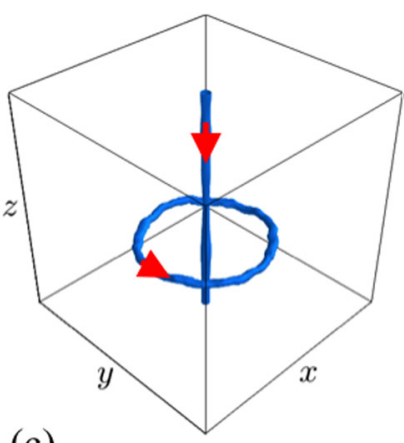

(c)

FIG. 1. (a) Initial condition at $t=0$ given by a planar vortex ring visualized by the isodensity tubular surface $\rho=0.1$, the arrow indicating vorticity direction. (b) Twist $T w=1$ is superposed by prescribing a full rotation of an isophase $\bar{\chi}$ as shown by the phase contour in the $(y, z)$ plane. (c) The presence of twist induces an instantaneous production of a new, central defect, here shown at time $t=20$ (adapted from Ref. [2]).

quantity of Euler equations classically defined by

$$
H=\int_{\Omega(\omega)} \mathbf{u} \cdot \omega d \mathbf{X}^{*}
$$

where $\Omega(\omega)$ denotes the domain of definition of vorticity, is by the Noether theorem also conserved in the GPE context. However, since vorticity is singular, $\Omega(\boldsymbol{\omega})$ has measure zero (in distributional sense), so that by definition of circulation and by Eq. (4) we have

$$
H=\Gamma \oint_{\mathcal{L}} \mathbf{u} \cdot d \mathbf{X}^{*}=\Gamma \oint_{\mathcal{L}} \nabla \chi \cdot d \mathbf{X}^{*}=0
$$

at all times, a result confirmed by several numerical experiments (see, for example, Refs. [1,9]). As for the classical case, the helicity of a collection of $N$ distinct vortex lines admits topological interpretation in terms of linking numbers [10,11], given by

$$
\mathcal{H}=\sum_{i \neq j} \Gamma_{i} \Gamma_{j} L k_{i j}+\sum_{i} \Gamma_{i}^{2} S L_{i}
$$

where $L k_{i j}$ is the (Gauss) linking number between $\mathcal{L}_{i}$ and $\mathcal{L}_{j}(i \neq j, i, j=1, \ldots, N)$ and $S L_{i}$ the (Călugăreanu-White) self-linking of each vortex. As shown by Salman [12] in his derivation of self-linking from helicity, the decomposition $S L_{i}=W r_{i}+T w_{i}$ in terms of writhe $W r_{i}$ and twist $T w_{i}$ of each vortex, holds true also for quantum defects. Zuccher and Ricca [1] showed that twist can indeed be identified with the rotation of the isophase surface around the nodal line. Recent work [2] has also shown that the superposition of twist on an existing defect [Fig. 1(a)] has the dramatic effect of producing a new, secondary phase defect in the system [Fig. 1(c)]. To elucidate how a localized twist disturbance can generate a new defect far afield in the system we shall consider the induced effect of phase twist perturbation on the fundamental state. As we shall see, the production of new defects by twist induction can be justified by hydrodynamic reasoning and explained by purely topological arguments. Since the new phase twist is a manifestation of a change of topology in the system, the production of a new defect can thus be interpreted as a result of a Aharonov-Bohm effect.

\section{TWIST DEFINITION AND HYDRODYNAMIC INTERPRETATION}

For a proper definition of twist in the GPE context let us introduce the mathematical ribbon $R\left(\mathbf{X}^{*}, \mathbf{X}^{+}\right)$given by the base curve $\mathcal{L}$ defined by $\mathbf{X}^{*}$ and its push-off $\mathbf{X}^{+}$on an isophase $\chi=\bar{\chi}=$ const. Let $(r, \theta, \xi)$ be local cylindrical coordinates centered on $\mathcal{L}$ with $\xi$ directed along $\hat{\mathbf{T}}, \bar{\chi}$ the isophase defined by a given value of $\theta$, and $\mathbf{X}^{+}=\mathbf{X}^{*}(s)+\varepsilon \hat{\mathbf{U}}(s)$; the ribbon width $\varepsilon$ is taken small with respect to the typical length scale of the defect and $\hat{\mathbf{U}}(s)$ is a unit vector normal to the defect and directed to a neighboring point on $\bar{\chi}$. The ribbon is then identified by the portion of $\bar{\chi}$ bounded by the defect and its $\varepsilon$ push-off $\mathbf{X}^{+}$. Total twist is standardly defined by the rate of rotation of $\hat{\mathbf{U}}$ around $\mathcal{L}$ integrated along the defect line, i.e.,

$$
T w=\frac{1}{2 \pi} \oint_{\mathcal{L}}\left(\hat{\mathbf{U}} \times \frac{d \hat{\mathbf{U}}}{d s}\right) \cdot \hat{\mathbf{T}} d s,
$$

and measures the total rotation of the isophase $\bar{\chi}$ around $\mathcal{L}$ (see also Ref. [12]). Twist admits decomposition in terms of normalized total torsion $T$ and intrinsic twist $\mathcal{N}$ [10], i.e.,

$$
T w=\frac{1}{2 \pi} \oint_{\mathcal{L}} \tau(s) d s+\frac{[\Theta]_{\mathcal{L}}}{2 \pi}=T+\mathcal{N},
$$

where $\tau(s)$ is the local torsion of $\mathcal{L},[\Theta]_{\mathcal{L}}$ the total rotation angle of $\hat{\mathbf{U}}$ around $\mathcal{L}$, and $[\Theta]_{\mathcal{L}} / 2 \pi=\mathcal{N}$ the number of full rotations. If $\mathcal{L}$ is a straight line of length $L$, then uniform twist is given by the uniform rotation of $\hat{\mathbf{U}}$ around $\mathcal{L}$ with $\mathcal{N} / L=m / L$ the number of full rotations per unit length [see Fig. 2(a)].

The physical effect of twist is made explicit by considering a stationary, straight defect $\mathcal{L}$ in isolation represented by the straight ribbon of Fig. 2(a), and the velocity interpretation of the phase gradient $\nabla \chi=\mathbf{u}$, decomposed locally in cylindrical polar components $\left(u_{r}, u_{\theta}, u_{\xi}\right)$. If the isophase surface $\bar{\chi}$ is not twisted it simply coincides with the coordinate plane $\theta=$ const, with gradient $\mathbf{u}_{\theta}=(\Gamma / 2 \pi r) \hat{\mathbf{e}}_{\theta}$ given by the classical rectilinear vortex solution. In this case the family of isophase surfaces hinged on a straight axis foliate the ambient space by a fan of $\theta$ planes (forming an "open-book decomposition"), with streamlines planar circles centered on the straight axis $\mathcal{L}$ and lying on concentric cylinders. When local twist is 


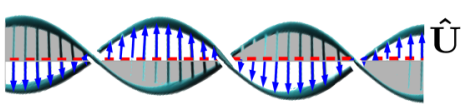

(a)

(c)

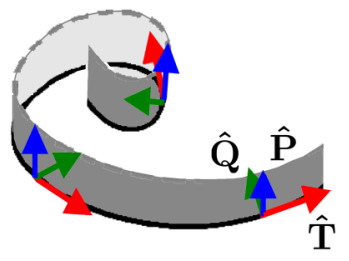

(b)

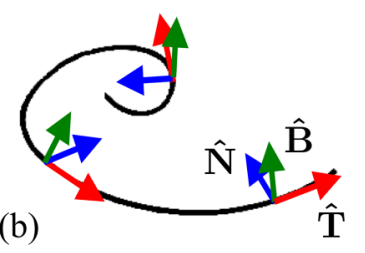

(d)

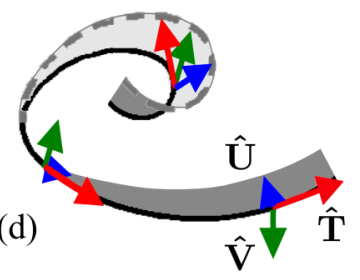

FIG. 2. (a) Uniform twist visualized by the uniform rotation of the ribbon spanwise unit vector $\hat{\mathbf{U}}$ around the ribbon straight axis. (b) Frenet frame $\{\hat{\mathbf{T}}, \hat{\mathbf{N}}, \hat{\mathbf{B}}\}$ on a base space curve (thick, black line); (c) parallel transport frame $\{\hat{\mathbf{T}}, \hat{\mathbf{P}}, \hat{\mathbf{Q}}\}$; (d) phase twist frame $\{\hat{\mathbf{T}}, \hat{\mathbf{U}}, \hat{\mathbf{V}}\}$.

different from zero the isophase surfaces are no longer planar, forming helical surfaces hinged on $\mathcal{L}$, now with a nonzero axial velocity $\mathbf{u}_{\xi}$ along $\mathcal{L}$.

It has been already observed that an $m$-phase perturbation acquired by a wave function of the fundamental state implies the generation of wavefront dislocations as a Aharonov-Bohm effect [13]. Hence the superposition of an $m$ phase on an $n$-star defect is expected to induce the production of a secondary singularity of strength $m$ [6]. This is exactly what has been observed in Ref. [2]. Since the $n$-star wavefronts are related to the action of an azimuthal velocity $\mathbf{u}_{\theta}$ around $\mathcal{L}$, similarly the induced twist due to an $m$-phase perturbation along $\mathcal{L}$ is associated with an axial velocity $\mathbf{u}_{\xi}$ along the defect; thus, the $n$-star wavefronts are led to rotate around the original defect and, as we shall demonstrate below, this gives rise to a secondary vortex of strength $m(\bmod 2 \pi)$. The corresponding additional axial velocity $\mathbf{u}_{\xi}$ changes the momentum from $\mathbf{P}$ to $\mathbf{P}+\mathbf{u}_{\xi}$, with the free part of the GPE Hamiltonian given (per unit of mass) by $H_{\text {free }}=\left(\nabla+\mathbf{u}_{\xi}\right)^{2} / 2$. This is in analogy with the Hamiltonian of an electron under the influence of a magnetic field, where $\mathbf{u}_{\xi}$ plays the role of the magnetic vector potential $\mathbf{A}$, as in the original Aharonov-Bohm experiment [14].

\section{PROOF OF TWIST EFFECTS AND PRODUCTION OF NEW DEFECT}

Here we determine the analytical condition for the presence of a phase twist and its physical consequence. First, we need to establish the pointwise condition for having (nonzero) twist on a nodal line through the ribbon spanwise unit vector $\hat{\mathbf{U}}$; second, by identifying a portion of isophase with the ribbon, we determine the local effect of twist in hydrodynamic terms; finally, by using topological arguments, we prove how new topological defects must emerge.

\section{A. Twist condition}

Since twist is locally detected by the rotation of $\hat{\mathbf{U}}$ around $\mathcal{L}$, we need to determine the twist condition in full generality, i.e. in a nonrotating, noninertial frame. This condition is provided by Fermi-Walker (FW) transport $[15,16]$. For a generic triad $\{\hat{\mathbf{T}}, \hat{\mathbf{P}}, \hat{\mathbf{Q}}\}(\hat{\mathbf{Q}}=\hat{\mathbf{T}} \times \hat{\mathbf{P}})$ on $\mathbf{X}^{*}, \mathrm{FW}$-transport of $\hat{\mathbf{P}}$ is governed by the equation

$$
\frac{D_{\mathrm{FW}} \hat{\mathbf{P}}}{D s}=\frac{d \hat{\mathbf{P}}}{d s}-(\hat{\mathbf{P}} \cdot \hat{\mathbf{T}}) \frac{d \hat{\mathbf{T}}}{d s}+\left(\hat{\mathbf{P}} \cdot \frac{d \hat{\mathbf{T}}}{d s}\right) \hat{\mathbf{T}} .
$$

Thus [see Fig. 2(c)]:

Definition 1 (Zero-twist condition): The unit vector $\hat{\mathbf{P}}$ does not rotate along $\mathcal{L}$ if and only if it is Fermi-Walker-transported along $\mathcal{L}$, that is,

$$
\frac{D_{\mathrm{FW}} \hat{\mathbf{P}}}{D s}=0, \quad \forall s \in \mathbf{X}^{*} .
$$

Let us consider the standard Frenet frame $\{\hat{\mathbf{T}}, \hat{\mathbf{N}}, \hat{\mathbf{B}}\}$ with $\hat{\mathbf{N}}$ and $\hat{\mathbf{B}}$ the usual normal and binormal unit vectors to $\mathbf{X}^{*}$ [see Fig. 2(b)] and apply Eq. (9) to the ribbon spanwise unit vector $\hat{\mathbf{U}}$. Using Frenet-Serret equations, with $c$ curvature of $\mathcal{L}$, a first computation gives

$$
\frac{D_{\mathrm{FW}} \hat{\mathbf{U}}}{D s}=\frac{d \hat{\mathbf{U}}}{d s}-c(\hat{\mathbf{B}} \times \hat{\mathbf{U}}) .
$$

Taking $\hat{\mathbf{U}}=\cos \theta(s) \hat{\mathbf{N}}+\sin \theta(s) \hat{\mathbf{B}}$ a second computation gives the pointwise twist condition for $\hat{\mathbf{U}}$ along $\mathcal{L}$, i.e.,

$$
\frac{D_{\mathrm{FW}} \hat{\mathbf{U}}}{D s}=\left(\tau+\theta^{\prime}\right) \hat{\mathbf{e}}_{\theta},
$$

where $\theta^{\prime}=d \theta / d s$ and $\hat{\mathbf{e}}_{\theta}$ the azimuthal unit vector around $\mathcal{L}$. This means that a ribbon is twisted iff $\hat{\mathbf{U}}$ rotates with rotation rate given by the sum of two contributions, $\tau$ due to the rotation of the Frenet-Serret triad with respect to the FW frame and $\theta^{\prime}$ given by the rotation of $\hat{\mathbf{U}}$ in the Frenet-Serret system. The isophase twist is thus determined by the twist condition (12) on $\hat{\mathbf{U}}$, made here explicit by the Fermi-Walker derivative.

Since the rotation of $\hat{\mathbf{U}}$ is also governed by the equation of rigid body rotation, we have

$$
\frac{D_{\mathrm{FW}} \hat{\mathbf{U}}}{D s}=\boldsymbol{\Omega}_{\xi} \times \hat{\mathbf{U}}=\Omega(\hat{\mathbf{T}} \times \hat{\mathbf{U}}) .
$$

Now locally - in a FW frame-we can identify the ribbon spanwise unit vector $\hat{\mathbf{U}}$ with the portion of the isophase surface close to the nodal line, given (in general) by $e^{i x}$; thus, from (12) and (13) we have

$$
\tau+\theta^{\prime}=\Omega=\frac{d \chi}{d s}=\nabla \chi \cdot \hat{\mathbf{T}},
$$

which identifies the rotation rate of the isophase (through $\hat{\mathbf{U}})$ with its tangential component along the nodal line. The velocity field interpretation of the phase twist rate given by Hänninen et al. (see their Eqs. (14) and (15) in Ref. [17]) is thus proved by Madelung transform.

\section{B. Twist effects}

If twist is superposed by a uniform twist rate of $2 \pi m / L$, then, using (13), we have

$$
\frac{D_{\mathrm{FW}} \hat{\mathbf{U}}}{D s}=\frac{2 \pi m}{L}(\hat{\mathbf{T}} \times \hat{\mathbf{U}})=\frac{2 \pi m}{L} \hat{\mathbf{e}}_{\theta} .
$$

By equating (12) with (15), we have a relation between local geometry and uniform twist, i.e., $\tau+\theta^{\prime}=2 \pi m / L$. Moreover, 

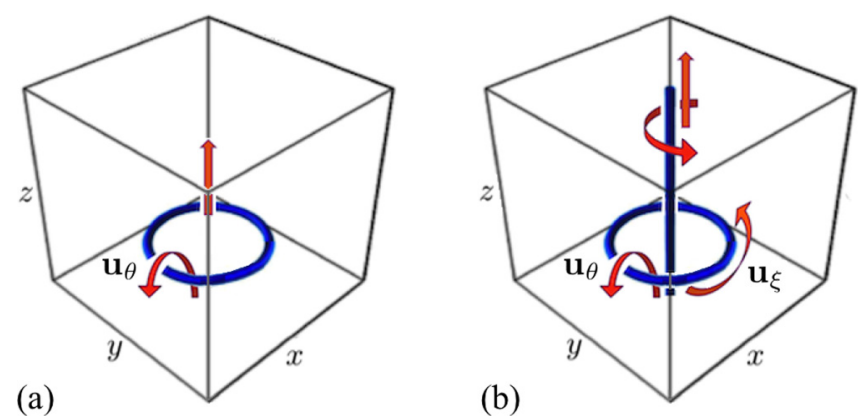

FIG. 3. Hydrodynamic interpretation of the phase twist on a vortex ring. (a) Generation of a secondary, central flow field without singularity by the action of a pure azimuthal velocity $\mathbf{u}_{\theta}$ on the vortex ring; (b) generation of a new singularity along the ring axis by the combined action of an azimuthal and an axial velocity field $\mathbf{u}_{\theta}$ and $\mathbf{u}_{\xi}$ along the ring centreline.

by equating (11) with (15), we can determine the standard rotation rate of $\hat{\mathbf{U}}$, given by

$$
\frac{d \hat{\mathbf{U}}}{d s}=\frac{2 \pi m}{L} \hat{\mathbf{e}}_{\theta}+c(\hat{\mathbf{B}} \times \hat{\mathbf{U}})=\frac{2 \pi m}{L} \hat{\mathbf{e}}_{\theta}-c \cos \theta \hat{\mathbf{T}} .
$$

Using the Madelung transform again, we can interpret the two contributions on the right-hand side above in terms of an azimuthal flow $\tilde{\mathbf{u}}_{\theta}=(2 \pi m / L) \hat{\mathbf{e}}_{\theta}$ around the defect line and an axial flow $\tilde{\mathbf{u}}_{\xi}=-c \cos \theta \hat{\mathbf{T}}$ along the line. This proves the hydrodynamic consequence of twist.

\section{Topological constraint}

Now let us turn our attention to the production of new defects. Proof of this is simply based on topological arguments. The simultaneous presence of the two terms above for the production of a new defect is a necessary topological condition. Indeed since the GPE helicity is always zero, by equating (5) to (6) (and setting circulations equal to 1) we must have that the sum of all linking numbers must be zero, too.

In the case of a single vortex ring in isolation exemplified by Fig. 3(a) we have $L k_{i j}=0$ and $S L_{1}=W r_{1}+T w_{1}=0$. However, if superposition of twist gives $T w_{1}=+1$ (with pure azimuthal flow), since for a plane circle $W r_{1}=0$, then we have a contradiction with the zero helicity requirement. Indeed, from (6), we have

$$
\mathcal{H} / \Gamma=S L_{1}=0 \neq W r_{1}+T w_{1}=+1 .
$$

The situation can only be rectified by the presence of a new, secondary defect (a straight vortex line in the center) with $W r_{2}=0$ and $T w_{2}=+1$, so that by (5) and (6) we have

$H=0=2 L k_{12}+T w_{1}+T w_{2}=2(-1)+1+1=0$.

It is this topological condition that makes the physical production of the new defect a manifestation of the Aharonov-Bohm effect.

\section{Production of new defect by twist induction}

As an example, consider the test case of Fig. 1(a), where $c=R^{-1}$ is the curvature of the vortex ring. The decomposition (16) that corresponds to the simultaneous generation of an azimuthal and an axial flow is essential to understand the formation of a new singularity on the central axis of the ring; using the Biot-Savart law (3) we evidently see how the induction of a pure azimuthal flow $\mathbf{u}_{\theta}$ [assuming for the moment $\mathbf{u}_{\xi}=0$; see Fig. 3(a)] can only generate pure axial flow along the central axis, without creating any circulation around it and hence no new defect. The production of a new defect can only take place when there is also circulation around the $z$ axis and this, by the Biot-Savart law, can be achieved when $\mathbf{u}_{\xi} \neq 0$ along the vortex ring as shown in Fig. 3(b). This is in good agreement with what has been observed in numerical experiments [2].

\section{TWIST INDUCTION BY PHASE PERTURBATION}

A direct relation between twist effects and phase perturbation is obtained by considering the superposition of a perturbation $\psi_{1}$ onto the GPE fundamental state $\psi_{0}$, i.e., $\psi=\psi_{0}+$ $\psi_{1}\left(\left|\psi_{1}\right| \ll\left|\psi_{0}\right| \ll 1\right)$. In absence of twist, perturbations are known to generate Kelvin waves. Indeed, by substituting the new $\psi$ into (2) and retaining linear terms in $\psi_{1}$, we recover the Schrödinger equation for $\psi_{1}$. With reference to the vortex ring case and by taking $(R, \phi, z)$ cylindrical coordinates centered on the ring central axis at $z=0$, we have

$$
\psi_{1}=\lambda e^{i(\boldsymbol{k} \cdot \boldsymbol{R}-v t)}, \quad|\lambda|=\text { const } \ll 1,
$$

where $v$ is the perturbation frequency, $\boldsymbol{k}$ the wave vector, and $\boldsymbol{R}$ the perturbed vector position. The dispersion relation for Kelvin waves is given by

$$
v=\frac{1}{2}\left(k^{2}-1\right)
$$

from which we can see that the group velocity $\nabla v$ is linear in $k=|\boldsymbol{k}|$.

Let us consider the presence of twist. This corresponds to superpose a shifted phase increment on the ground-state wave. Let $w=\theta / \phi$ be the winding number given by the ratio of poloidal to toroidal angles. The superposition of total twist through $w$ full rotations on the ring defect (no torsion) generates a new unperturbed state:

$$
\psi_{0} \longrightarrow \psi_{0} e^{i w \phi}, \quad w \in \mathbb{Z} .
$$

Since GPE is not locally phase invariant, we get a new equation where the phase twist plays the role of a new potential, i.e.,

$$
\frac{\partial \psi_{0}}{\partial t}=\frac{i}{2}\left(\nabla+\frac{i w}{R} \hat{\mathbf{e}}_{\phi}\right)^{2} \psi_{0}+\frac{i}{2}\left(1-\left|\psi_{0}\right|^{2}\right) \psi_{0} .
$$

By perturbing this new, fundamental state, we have

$$
\psi=e^{i w \phi} \psi_{0}+\lambda e^{i(\boldsymbol{k} \cdot \boldsymbol{R}-v t)}, \quad|\lambda|=\mathrm{const} \ll 1 ;
$$

and by linearizing we obtain the new equation for $\psi_{1}$, given by

$$
\frac{\partial \psi_{1}}{\partial t}=\frac{i}{2}\left(\nabla+\frac{i w}{R} \hat{\mathbf{e}}_{\phi}\right)^{2} \psi_{1}+\frac{i}{2} \psi_{1} .
$$

This equation is very similar to that of AharanovBohm $[13,14,18]$, where the additional term $(i w / R) \hat{\mathbf{e}}_{\phi}$ plays the role of the vector potential created by a singular magnetic field at the origin. Note that $(w / R) \hat{\mathbf{e}}_{\phi}=(\Gamma / 2 \pi R) \hat{\mathbf{e}}_{\phi}$ can indeed be interpreted as the velocity field induced by the central straight vortex. By substituting the plane-wave form of $\psi_{1}$ and 
considering steady conditions in cylindrical coordinates, we obtain the new dispersion relation

$$
v=\frac{1}{2}\left(\boldsymbol{k}+\frac{w}{R} \hat{\mathbf{e}}_{\phi}\right)^{2}-\frac{1}{2},
$$

where now the group velocity depends linearly also on $w$. Note that the same result can be obtained by direct perturbation of the fundamental state governed by the standard GPE (2) through a linear perturbation that encapsulates also a phase-shift contribution.

\section{VORTEX DEFECTS IN BOSE-EINSTEIN CONDENSATES}

\section{A. Production and manipulation of BECs defects}

Bose-Einstein condensates are usually created and manipulated by trapping and cooling atoms (typically ${ }^{23} \mathrm{Na}$ or ${ }^{87} \mathrm{Rb}$ ) by magnetic devices and laser techniques. Magnetic traps [19] are more efficient than laser cooling and are realized by combining two magnetic fields, one uniform in a given direction (say along $z$ ) and one (due to quadrupole magnets) harmonic and perpendicular to the former. The resulting field has thus a minimum at the center. Since particles with high magnetic moments have minimum energy in low-magneticfield regions, only these particles (the so-called low field seekers) get trapped at the center, while the others (the high field seekers) are expelled from the trap, hence cooling down the system.

There are many different methods to create and visualize vortex defects in BECs [20-24]. One earlier, ingenious method to imprint vortices [25] is to induce a Berry phase onto the atoms of the condensate. This is done by inverting the $z$ component of the magnetic field of a Ioffe-Pritchard magnetic trap and by doing so induce a rotation of the atoms' angular momentum (see Ref. [25] for technical details). To visualize and reconstruct vortex dynamics one can then use the recent technique proposed by Serafini et al. [26] by collecting a series of laser scans of the BECs sample.

A fundamental technique to manipulate BECs defects is based on the use of optical tweezers given by laser beams. A common method to transfer angular momentum and spin to defects is by using Gauss-Laguerre beams [27]. Polarization of the beam modifies the spin state of the atoms, while orbital angular momentum (OAM) modifies the phase. A GaussLaguerre beam in the state $L G_{p}^{w}$ is characterized by two integers $p$ and $w: p+1$ denotes the number of nodes of the beam and $w$ the winding number. So, in general, $L G_{p}^{w}$ beams can transfer an azimuthal phase $e^{i w \phi}$ to BECs. An example of an $L G_{0}^{1}$ transfer is that given in Ref. [21], taking

$$
L G_{0}^{1}=\frac{2}{\sqrt{\pi}} \frac{r_{0}}{\delta_{1}^{2}} e^{-\frac{r_{0}^{2}}{\delta_{1}^{2}}} e^{i \phi},
$$

where $r_{0}$ is the radius of the beam from its center (with singularity at $r_{0}=0$ ) and $\sqrt{2} \delta_{1}$ is the peak-to-peak beam diameter. This mode transfers an OAM of $\hbar$ units to each atom of the defect. A singularity is thus created by a Raman transition, exciting the atoms hit by the beam to acquire a higher spin state.

\section{B. Proposed experiment}

To create a vortex ring with phase twist we propose to use a toroidal trap as in Ref. [28] and injection of twist by the Berry phase technique used in Ref. [25]. First, the orthogonal quadrupole field for the magnetic trap must be modified in order to produce a variation of the rotation axis of the atoms' angular momentum along the toroidal direction $\phi$ of the trap (see Ref. [25] for details). This can be achieved by prescribing a field with twist given by a combination of toroidal and poloidal components as in Ref. [29]. By doing so we will imprint the generation of a vortex ring defect and, simultaneously, a superposed phase twist given by the rotation axis of the atoms' angular momentum. This rotation will now be subject to the combined effects along the toroidal direction $\phi$ and the poloidal direction $\theta$, responsible for the phase twist along $\phi$. As a result the induced velocity will have a toroidal component along the vortex ring (absent in the case of zero phase twist) that will generate the straight vortex defect at the center of the torus. By perturbing the vortex ring thus generated one can then measure the Kelvin waves propagation as done in [22] and compare the dispersion relation with our Eq. (24).

An alternative method to generate phase twist is to use $L G_{p}^{w}$ beams to induce an OAM on the atoms near the torus nodal line. The defect phase should then acquire a winding number around the nodal line induced by the $L G_{p}^{w}$ beam. Again, by measuring the dispersion relation one can get information on the group and phase velocity of the perturbation, and by Eq. (24) determine the relationship between the wave frequency and the winding number $w$ of the $L G_{p}^{w}$ beam. A secondary straight defect of topological charge proportional to $w$ (in units of circulation) should appear at the center of the vortex ring. Since this will presumably be dynamically unstable [30], we expect its decay to a number of singly charged defects, orderly self-organized in the central region of the trap. We conjecture that if we make interfere a BEC in a rotational "twisted" state with a nonrotational (or counterrotating) state, the interference is likely to produce a forklike pattern identical to that observed in the standard AharonovBohm effect. In reconstructing the images from the relative interference patterns the two effects will be presumably indistinguishable.

\section{ACKNOWLEDGMENTS}

We gratefully acknowledge the input received from Iacopo Carusotto for reading a preliminary version of this paper and for his suggestions on the last section of this paper.
[1] S. Zuccher and R. L. Ricca, Relaxation of twist helicity in the cascade process of linked quantum vortices, Phys. Rev. E 95, 053109 (2017).
[2] S. Zuccher and R. L. Ricca, Twist effects in quantum vortices and phase defects, Fluid Dyn. Res. 50, 1 (2018). 
[3] E. P. Gross, Hydrodynamics of a superfluid condensate, J. Math. Phys. 4, 195 (1963).

[4] L. P. Pitaevskii, Vortex lines in an imperfect Bose gas, J. Exptl. Theoret. Phys. (U.S.S.R.) 40, 646 (1961) [Sov. Phys. JETP 13, 451 (1961)].

[5] C. F. Barenghi and N. G. Parker, A Primer on Quantum Fluids (Springer-Verlag, Berlin, 2016).

[6] M. V. Berry and M. R. Dennis, Knotted and linked phase singularities in monochromatic waves, Proc. R. Soc. Lond. A 457, 2251 (2001).

[7] A. T. Winfree and S. H. Strogatz, Singular filaments organize chemical waves in three dimensions, Physica D 13, 221 (1984).

[8] F. Lund, Defect dynamics for the nonlinear Schrödinger equation derived from a variational principle, Phys. Lett. A 159, 245 (1991).

[9] S. Zuccher and R. L. Ricca, Helicity conservation under quantum reconnection of vortex rings, Phys. Rev. E 92, 061001 (2015).

[10] H. K. Moffatt and R. L. Ricca, Helicity and the Călugăreanu invariant, Proc. R. Soc. Lond. A 439, 411 (1992).

[11] R. L. Ricca, Applications of knot theory in fluid mechanics, in Knot Theory, edited by V. F. R. Jones et al., Vol. 42 (Polish Academy of Sciences; Institute of Mathematics: Banach Center Publications, 1998), pp. 321-346.

[12] H. Salman, Helicity conservation and twisted Seifert surfaces for superfluid vortices, Proc. R. Soc. A 473, 20160853 (2017).

[13] M. V. Berry, R. G. Chambers, M. D. Large, C. Upstill, and J. C. Walmsley, Wavefront dislocations in the Aharonov-Bohm effect and its water wave analog, Eur. J. Phys. 1, 154 (1980).

[14] B. Aharonov and D. Bohm, Significance of the electromagnetic potentials in the quantum theory, Phys. Rev. 115, 485 (1959).

[15] J. L. Synge, Relativity, The General Theory (North-Holland, Amsterdam, 1960).

[16] R. L. Bishop, There is more than one way to frame a curve, Am Math. Mon. 82, 246 (1975).

[17] R. Hänninen, N. Hietala, and H. Salman, Helicity within the vortex filament model, Sci. Rep. 6, 37571 (2016).

[18] E. Sonin, Magnus force, Aharonov-Bohm effect, and Berry phase in superfluids, in Quantized Vortex Dynamics and Superfluid Turbulence, edited by C. F. Barenghi, R. J. Donnelly, and W. F. Vinen, Lecture Notes in Physics Vol. 571 (SpringerVerlag, Berlin, 2001), pp. 131-137.
[19] D. E. Pritchard, Cooling Neutral Atoms in a Magnetic Trap for Precision Spectroscopy, Phys. Rev. Lett. 51, 1336 (1983).

[20] J. F. S. Brachmann, W. S. Bakr, J. Gillen, A. Peng, and M. Greiner, Inducing vortices in a Bose-Einstein condensate using holographically produced light beams, Opt. Express 19, 12984 (2011).

[21] J. P. Torres and L. Torner, Twisted Photons. Applications of Light with Orbital Angular Momentum (Wiley, New York, 2011).

[22] E. Fonda, D. P. Meichle, N. T. Ouellette, S. Hormoz, and D. P. Lathrop, Direct observation of Kelvin waves excited by quantized vortex reconnection, Proc. Natl. Acad. Sci. USA 111, 4707 (2014).

[23] L. Dominici, R. Carretero-González, A. Gianfrate, J. CuevasMaraver, A. S. Rodrigues, D. J. Frantzeskakis, G. Lerario, D. Ballarini, M. De Giorgi, G. Gigli, P. G. Kevrekidis, and D. Sanvitto, Interactions and scattering of quantum vortices in a polariton fluid, Nat. Commun. 9, 1467 (2018).

[24] A. Gianfrate, L. Dominici, O. Voronych, M. Matuszewski, M. Stobińska, D. Ballarini, M. De Giorgi, G. Gigli, and D. Sanvitto, Superluminal X-waves in a polariton quantum fluid, Light Sci. Appl. 7, 17119 (2018).

[25] A. E. Leanhardt, A. Görlitz, A. P. Chikkatur, D. Kielpinski, Y. Shin, D. E. Pritchard, and W. Ketterle, Imprinting Vortices in a Bose-Einstein Condensate using Topological Phases, Phys. Rev. Lett. 89, 190403 (2002).

[26] S. Serafini, L. Galantucci, E. Iseni, T. Bienaimé, R. N. Bisset, C. F. Barenghi, F. Dalfovo, G. Lamporesi, and G. Ferrari, Vortex Reconnections and Rebounds in Trapped Atomic Bose-Einstein Condensates, Phys. Rev. X 7, 021031 (2017).

[27] S. J. Parkin, T. A. Nieminen, N. R. Heckenberg, and H. Rubinsztein-Dunlop, Optical measurement of torque exerted on an elongated object by a noncircular laser beam, Phys. Rev. A 70, 023816 (2004).

[28] C. Ryu, M. F. Andersen, P. Cladé, V. Natarajan, K. Helmerson, and W. D. Phillips, Observation of Persistent Flow of a BoseEinstein Condensate in a Toroidal Trap, Phys. Rev. Lett. 99, 260401 (2007).

[29] F. Maggioni and R. L. Ricca, On the groundstate energy of tight knots, Proc. R. Soc. A 465, 2761 (2009).

[30] L. Giacomelli and I. Carusotto, Ergoregion instabilities and vortex stability in dilute Bose-Einstein condensates, arXiv: $1905.02447 \mathrm{v} 2$ [cond-mat.quant-gas]. 